Review

\title{
Biochemical and Genetic Engineering of Diatoms for Polyunsaturated Fatty Acid Biosynthesis
}

\section{Hong-Ye Li *, Yang Lu, Jian-Wei Zheng, Wei-Dong Yang and Jie-Sheng Liu *}

Key Laboratory of Eutrophication and Red Tide Prevention of Guangdong Higher Education Institutes, Jinan University, Guangzhou 510632, China; E-Mails: biglvyangaa@gmail.com (Y.L.); jwzheng89@126.com (J.-W.Z.); tywd@jnu.edu.cn (W.-D.Y.)

* Authors to whom correspondence should be addressed; E-Mails: thyli@jnu.edu.cn (H.-Y.L.); 1js@jnu.edu.cn (J.-S.L.); Tel.: +86-20-85228470 (H.-Y.L.); Tel.: +86-20-85220006 (J.-S.L.).

Received: 18 October 2013; in revised form: 10 December 2013 / Accepted: 12 December 2013 / Published: 7 January 2014

\begin{abstract}
The role of diatoms as a source of bioactive compounds has been recently explored. Diatom cells store a high amount of fatty acids, especially certain polyunsaturated fatty acids (PUFAs). However, many aspects of diatom metabolism and the production of PUFAs remain unclear. This review describes a number of technical strategies, such as modulation of environmental factors (temperature, light, chemical composition of culture medium) and culture methods, to influence the content of PUFAs in diatoms. Genetic engineering, a newly emerging field, also plays an important role in controlling the synthesis of fatty acids in marine microalgae. Several key points in the biosynthetic pathway of PUFAs in diatoms as well as recent progresses are also a critical part and are summarized here.
\end{abstract}

Keywords: diatom; fatty acids; PUFA; genetic engineering

\section{Introduction}

Marine diatoms have been explored recently as sources of secondary metabolites with biological activity. Diatoms are known to be a reserve source for biofuel, marine drugs, and live feed, owing to their short life cycle, fast growth and simple nutritional requirements [1,2]. Marine diatoms contribute to approximately $40 \%$ of primary productivity in marine ecosystems and $20 \%$ of global carbon fixation [1,3]. Diatoms are not only photosynthetic autotrophs but can also be symbiotic with 
photosynthetic organisms [4,5]. Diatoms are considered to be highly diverse, with an estimated $10^{5}$ to $10^{7}$ species, more than any other single algal group [6,7]. A defining characteristic of diatoms is the frustule, which is composed of hydrated silicon dioxide and organic materials; environmental factors play a critical part in driving the morphological variation of frustules [8]. Diatom chloroplasts contain chlorophyll $a, c 1$, and $c 2$. The golden brown fucoxanthin, a promising antioxidant, anti-inflammatory drug, and anticancer drug, is a major carotenoid of diatoms [9]. Diatoms have been attracting increasing interest for their biological characteristics and application value. Due to their quantity, industrial scale culture conditions, as well as socio-economic considerations, diatoms are one of the most suitable raw materials for the production of bioactive metabolites. Progress on the identification of all of these components has recently been facilitated due to the development of new techniques, such as molecular genetic tools and different chemical approaches. However, aspects of diatom metabolism and the production of natural compounds still remain largely unknown.

\section{Polyunsaturated Fatty Acids (PUFAs) and the Diatom}

Certain indispensable fatty acids are important as components of the cell membrane, lipid storage or signal transduction pathways, for instance, polyunsaturated fatty acids (PUFAs). PUFAs are approximately 18-22 carbon, straight-chain fatty acids that contain two or more double bonds. PUFAs can mainly be classified into two principal families: $n-6$ (or $\omega-6$ ) and $n-3$ (or $\omega-3$ ) families that are derived biosynthetically from linoleic acids (18:2n-2) and $\alpha$-linolenic acids (18:3n-3), respectively, and these two essential fatty acids (EFA) are crucial for human health. In the two families, $n-3$ PUFAs are represented by $\alpha$-linolenic acid (ALA, 18:3n-3), stearidonic acid (SDA, 18:4n-3), eicosapentaenoic acid (EPA, 20:5n-3) and docosahexaenoic acid (DHA, 22:6n-3); n-6 PUFAs are represented by arachidonic acid (AA, 20:4n-6) and $\gamma$-linoleic acid (GLA, 18:3n-6). PUFAs as bioactive substances, especially the $n-3$ series, are essential nutrients that promote human health and growth in animals [10]. EPA and DHA play a favorable role in the cardiovascular system [11], vision [12], and treating psychiatric disorders [13]; while C18n-3 PUFAs do not confer such health benefits. Studies have shown that larger ratios of $n-6$ to $n$-3 PUFAs correlate with the increased pathogenesis of many diseases, including coronary heart disease (CHD) $[14,15]$. A convincing study by WHO/FAO demonstrated that consuming $250 \mathrm{mg}$ (primary prevention) to $2 \mathrm{~g}$ (secondary prevention) of EPA and DHA every day can prevent CHD and reduce the risk of fatal CHD events (reviewed by Martins [16]). For human nutrition, DHA, an essential fatty acid, plays an important role in preventing age-associated declines in cognition, such as Alzheimer's disease, multiple sclerosis and Parkinson's disease. DHA is also an active ingredient for the treatment of psychiatric conditions due to its function in nervous system development [17]. It must be emphasized that DHA can promote visual system development to enhance retinal function and visual acuity; particularly, preterm infants can benefit from an extra DHA supplement [18]. In aquaculture, EPA and DHA are the key nutritional constituents of the larvae of many fish, shrimp and bivalve organisms. PUFAs, which have the potential to maintain a high larval growth rate and high reproductive rate, are known in a wide manifold of marine and freshwater organisms [19-21]. Thus, PUFA content is one of the most important indices for the evaluation of aquatic feed nutritional value.

Due to the benefits of PUFAs in humans and animals, a large amount of PUFA supplementation is needed. However, the shortage of PUFA biological resources has always restricted their wide 
application; inexpensive DHA and EPA biological resources have become an urgent demand. It is generally recognized that the main sources of PUFAs on the market are marine fish because there are no reported definitive examples of land plants accumulating EPA and DHA [16]. Although the content of DHA and EPA in fish oil can reach approximately $20 \%-30 \%$, there are many issues that remain to be solved. It must be emphasized that the quality of fish oil can vary much under different circumstances, for instance, fatty fish stemming from contaminated regions, those obtained during the fishing season, or the species of fish [22]. In addition, fish oil presents a disadvantage for some consumers due to its distinctive odor or off-favors that originate from lipid peroxidation [23].

Awareness of the benefits of PUFAs and the shortage of fish oil have led to the exploitation of new resources of PUFAs over the last few years. In actuality, fish are not real PUFA producers. Fish accumulate PUFAs through the ingestion of PUFA-rich microalgae. Microalgae, especially those of marine origin, as emerging resources of long-chain PUFAs, have caused increasing concern [24]. Lipid-rich microalgae have been regarded as promising candidates for the production of biofuels [25]. Diatoms are metabolically versatile species owing to the fact that they can produce and accumulate a range of bioactive metabolites, such as PUFAs and extracellular polymeric substances [1,26]. Using diatoms to produce PUFAs has more advantages than extraction from fatty fish. First, diatoms have a high content of PUFAs; the relative content of PUFAs in some species can reach up to $5 \%-6 \%$ of cell dry weight. Second, diatoms are single-celled microalgae with fast growth, and it is feasible to manipulate their metabolism to promote the synthesis of PUFAs using biotechnological means, such as genetic manipulation and modified culture conditions [27]. Thus, continuous mass production of diatoms using photobioreactors is easy, and highly stabilized metabolites can be obtained through controlled production conditions. Third, the extraction and purification of PUFAs from diatoms is much simpler than that from fatty fish [16]. In particular, diatom products do not contain a fishy smell, cholesterol, or pollution by pesticides and heavy metals that most likely appear in fish oil.

\section{Environmental Factors Controlling the Synthesis of PUFAs}

\subsection{Light}

More recently, microalgae biotechnologies have been explored intensively with the aim to increase fatty acid production. Light, one of the most important environmental factors of the marine ecosystem, is of increasing interest to modulate the growth and accumulation of fatty acids in microalgae. Microalgae biomass, fatty acid composition, and pigment and ester concentration are reported to be light sensitive, thus, much attention has been paid to enhancement of fatty acid production using variable light. Light, the source of energy for photosynthesis, can alter photosynthesis products to synthesize metabolites. The spectral property of light acts as a major factor in controlling photosynthetic efficiency. Blue and red light spectra have been revealed to be the most active for photosynthesis [28,29]. Studies of the effects of light on model diatom Phaeodactylum tricornutum suggested that $P$. tricornutum cultivated in tubular photobioreactors had decreasing biomass productivity under lowered light, whereas the EPA content showed increased production. Additionally, photo-inhibition, the phenomenon of excessively high irradiance on a culture, causes lower pigment content [30]. Studies on other microalgae also confirmed the correlation between cellular metabolism and light effects. For instance, Piepho et al. found that the 
interactive effects of light and phosphorus supply were most pronounced in the diatom Cyclotella meneghiniana, compared with the other two green algae Scenedesmus quadricauda and Chlamydomonas globosa, and a cryptophyte Cryptomonas ovata, and hypothesized that increasing light intensity can lead to higher production of total fatty acids (TFAs), saturated fatty acids (SFAs), and monounsaturated fatty acids (MUFAs), both at low phosphorus concentrations and high phosphorus concentrations in the centric diatom Cyclotella meneghiniana [31]. More distinct changes in several SFA and UFA (unsaturated fatty acid) concentrations with light were found in the low-P treatments compared with the high-P treatments. It is worth mentioning that in Cryptomonas ovata (Cryptophyceae), light intensity showed no effects on the production of TFAs, SFAs, MUFAs, and PUFAs [31]. In the range of maximum EPA productivity using P. tricornutum in a flat panel airlift reactor, photosynthesis efficiency reached $10.6 \%$ at a low light intensity $\left(250 \mu \mathrm{mol}\right.$ photon $\left.\mathrm{m}^{-2} \mathrm{~s}^{-1}\right)$ [32]. Studies on stream periphyton provide some explanation regarding the changing of PUFAs production. These studies showed that the proportion of PUFAs decreased with increasing light intensity and increased with phosphorus enrichment, opposite of that observed for SFA and MUFA. In particular, under high phosphorus treatment, there was a significant augmentation of DHA. A decline in ALA under high light intensity was accompanied by an increase in linoleic acid under increasing light, while arachidonic acid was hardly affected by either light or phosphorus supply [33].

\subsection{Temperature}

Temperature plays a critical role in cell growth and metabolite synthesis. As the growth temperature changes, the responses from different species show inconsistent relationships between temperature and percentage of unsaturated fatty acid [34-36]. The optimum growth temperatures for some microalgae have been determined to range from $16{ }^{\circ} \mathrm{C}$ to $27{ }^{\circ} \mathrm{C}$ [28]; for example, the optimum temperature for maximum growth rate of $P$. tricornutum is $20^{\circ} \mathrm{C}$. The growth response of $P$. tricornutum was hardly affected by temperatures in short-term treatments, but in long-term temperature treatments, a gradual decrease of growth at lower temperatures and a sharp drop of growth rate at higher temperatures occurred [37]. The temperature experiment conducted on Cyclotella meneghiniana revealed that, TFA, SAFA, and MUFA concentrations increased at $25^{\circ} \mathrm{C}$ compared to $10^{\circ} \mathrm{C}$ under low $\mathrm{P}$ supply; while no difference was seen between $10{ }^{\circ} \mathrm{C}$ and $25{ }^{\circ} \mathrm{C}$ in the high-P treatment [31]. With regard to Nitzschia closterium, which has a curved relationship between temperature and PUFA content, its optimum temperature is $20-30{ }^{\circ} \mathrm{C}$ [34]. Marine benthic diatom species, separated from the intertidal environment, could accumulate PUFAs during low-temperature treatment, while short-chain fatty acids, glycerol and glucose were accumulated in response to high-temperature treatments [38]. The results are consistent with the observations in Nitzschia frustulum (Kützing) where a decreasing production of saturated and monounsaturated fatty acids and an increasing production of fatty acids with a high degree of unsaturation were detected at a low temperature treatment $\left(10^{\circ} \mathrm{C}\right)[34]$, which plays a critical role in the maintenance of membrane fluidity [39]. In P. tricornutum, which is considered to be a promising candidate for biodiesel production and is characterized by high fatty acid content, the contents of EPA and PUFAs were found to be increased by up to $120 \%$ compared with the control when temperature was lowered from $25{ }^{\circ} \mathrm{C}$ to $10{ }^{\circ} \mathrm{C}$ for $12 \mathrm{~h}$, with PUFA and EPA yields of up to $4.9 \%$ and $2.6 \%$, respectively [40]. 


\subsection{Chemical Composition of Culture Medium}

Diatoms are able to rapidly adapt to the changing nutrient conditions. Particularly, in upwelling water environments where nutrients are brought to the surface, diatoms exhibit notable efficiency in the uptake of growth-limiting nutrients such as iron, nitrogen and silica [41,42]. The chemical composition of culture medium (nitrogen, phosphorus, iron, silicon salt, vitamins) has considerable importance on the composition and content of fatty acids.

The type of nitrogen source can also affect fatty acid content and growth of microalgae. Diatoms are able to utilize a variety of nitrogen sources, including inorganic $\left(\mathrm{NO}_{3}{ }^{-}, \mathrm{NH}_{4}{ }^{+}\right)$and organic (urea, amino acids) nitrogen, thereby adapting their nitrogen metabolism based on the available nutrients $[43,44]$. Among three nitrogen sources, $\mathrm{NaNO}_{3}$, urea, and $\mathrm{NH}_{4} \mathrm{Cl}$, tested in a lipid- and PUFA-rich pennate diatom Cylindrotheca species, the best growth of $C$. fusiformis occurred on nitrate and urea, while $\mathrm{NH}_{4} \mathrm{Cl}$ was best for $C$. closterium [45]. Lipid productivity was much higher in cultures supplied with $\mathrm{NH}_{4} \mathrm{Cl}$ for both $C$. fusiformis and C. closterium and compensated for the lower biomass in C. fusiformis. Thus, urea and $\mathrm{NaNO}_{3}$ are optimum nitrogen sources for growth of $C$. fusiformis, while $\mathrm{NH}_{4} \mathrm{Cl}$ is the best for $C$. closterium. The highest lipid content was achieved in both Cylindrotheca species in culture medium with $\mathrm{NH}_{4} \mathrm{Cl}$ as the nitrogen source. However, C. fusiformis showed a significantly lower growth rate in culture medium with $\mathrm{NH}_{4} \mathrm{Cl}$ than in medium with $\mathrm{NaNO}_{3}$ or urea [45]. Nitrate and urea are better than ammonium salts as nitrogen sources in P. tricornutum UTEX 640, a suitable strain for industrial production of EPA [32]. By using a flat panel airlift reactor, P. tricornutum biomass productivity reached $2.35 \mathrm{~g} \mathrm{~L}^{-1}$ day $^{-1}$ on urea at an aeration rate of $0.66 \mathrm{vvm}$ at continuous light supply (1000 $\mu \mathrm{mol}$ photon $\mathrm{m}^{-2} \mathrm{~s}^{-1}$ ), while productivity on nitrate never reached $1.37 \mathrm{~g} \mathrm{~L}^{-1} \mathrm{day}^{-1}$ [32]. Many microalgae species present a higher lipid content under nitrogen starvation conditions, including diatom P. tricornutum, green algae Chlorella spp., Botryococcus braunii, Chlamydomonas reinhardtii, and Dunaliella salina [46-50]. Nitrogen deprivation leads to a redirection of intracellular carbon flux, and the carbon source is no longer converted to cellular building blocks but instead funneled into triacylglycerol synthesis in P. tricornutum [51].

Iron, a vital component of the photosynthetic apparatus and mitochondrial electron transport chain, is a growth-limiting nutrient for photosynthetic microalgae. Iron limitation was found to lead to reduced synthesis of chlorophyll and a significant decrease in photosynthetic efficiency as well as slower nitrogen assimilation in diatoms $[52,53]$. Vitamin $\mathrm{B}_{12}$ (cobalamin) availability is evidenced to influence diatom growth. The abundance and wide distribution of transcripts of CBA1, a recently characterized cobalamin acquisition protein, in environmental samples, suggests that vitamin $\mathrm{B}_{12}$ is an important nutritional factor [54].

In the weakly silicified diatom P. tricornutum, increasing the concentration of silicate was found to cause a decrease in the content of EPA [32]. In the marine diatom Chaetoceros gracilis, the content of EPA and DHA decreased as silicate availability was reduced [55].

\subsection{Growth Stage and PUFA Accumulation}

Usually, slow growth of microalgae leads to a high proportion of storage lipids, while higher growth rates result in higher fractions of structural lipids. PUFAs as parts of structural biomolecules are 
essential components of cell membranes. At low growth rates, microalgae tend to accumulate neutral lipids rich in SFA (14:0, 16:0) and MUFA (16:1). These lipids are used to store the excess carbon accumulated during photosynthesis. On the contrary, at high growth rates, the demand for structural components increases, thereby reducing the surplus for neutral lipid accumulation and consequently increasing the proportion of PUFAs [56].

Cylindrotheca strains, which are not usually used as food for marine organisms, accumulate a high percentage of PUFAs at the exponential phase (29.5\%-42.9\%), especially 20:5 (n-3) and 20:4 (n-6) [57]. A study on four strains of Cylindrotheca indicated that the total lipid content reached its highest values in the late stationary phase of the culture, while PUFAs decreased in the later phase and peaked in the exponential phase or in the early stationary phase [57]. This result is consistent with that of diatom Thalassiosira pseudonana by Brown et al. [58].

\section{Growth Facilities}

Growth facilities of diatoms can be set up in aquatic areas, which would not cause an arable land crisis. High-efficiency photobioreactors are required to culture and tap the potential of microalgae [59]. Photobioreactor systems mainly consist of two types: open and closed systems.

Open systems for the culture of microalgae, including open ponds and raceways, have been well developed. These open systems possess major advantages, including being of low cost and having simple technical requirements. Open systems can be used for fast-growing species or for species that can be maintained at extreme conditions, such as high salinity or high $\mathrm{pH}$ conditions, in which contamination can be controlled. A number of microalgae species have been commercially cultivated at large scale with open systems, such as green algae Haematococcus pluvialis, blue-green alga Spirulina platensis, etc. However, some issues need to be addressed, such as the likelihood of contamination from unwanted algae and certain micro-organisms, significant evaporation losses, poor utilization of $\mathrm{CO}_{2}$ due to evaporation or stripping, poor thermal or temperature control, and inability to achieve high-density cultures due to poor mixing and optically dark zones.

Closed photobioreactors have attracted much attention because they allow for better control of the culture conditions than open systems, thereby providing robust culture systems for the culture of a large number of microalgae species [60]. Higher biomass productivities can be achieved and contamination can be easily reduced by using closed photobioreactors [59]. The available photobioreactors of closed systems may be classified into tubular reactors and panel reactors. These can be further categorized according to the device used for light supply, the arrangement of the growth units, the orientation of tubes or panels, the method of gas exchange system, the method of culture circulation, and the materials used for photobioreactor construction [61]. Among these categories, irrespective of the specific reactor configuration, several criteria need to be addressed, including effective and efficient light supply; $\mathrm{CO}_{2}$ supply with minimal losses; continuous removal of oxygen generated by photosynthesis, as excessive oxygen inhibits photosynthesis and metabolism; and good scalability of the photobioreactor technology [62].

In both outdoor and indoor cultivation systems, light source and light intensity are key factors affecting the phototrophic growth of microalgae [63]. Despite the innovation of a large number of photobioreactors, only a few of them can effectively utilize solar energy for mass cultivation of 
microalgae [59]. Sunlight is the major light source for outdoor cultivation systems; innovative electric light sources, such as light emitting diodes (LED) and optical fiber, are of interest for indoor cultivation systems and $24 \mathrm{~h}$ /day production [64]. LED has high electrical-to-optical power conversion efficiency although its infrastructure investment is a bit more expensive. Most outdoor reactors are designed to have largely exposed illumination surface areas. In this respect, flat panel, inclined and horizontal tubular reactors are promising, except for their weak scalability. Reactors such as bubble-column, airlift, and stirred-tank are easy to scale up, although their use in outdoor cultivation is limited due to their small illumination surface area [59].

\section{Genetic Engineering of Diatoms}

Although large-scale cultivation facilities for microalgae are available, their productivity is hampered by the lack of ideal strains that can be selectively optimized for both high biomass productivity and high TAG (triacylglycerol) or PUFA content [25]. A promising approach is the use of genetic engineering to substantially improve the diatom strains to produce PUFA. Genetically modified crops, mammals, and microalgae are emerging as alternative sources of PUFA [65,66]. Genetically engineered microalgae represent progress toward the use of fermentation approaches to commercially exploit microalgae at large scale, thereby reducing the limitations associated with photobioreactors such as light-dependency and lower growth [67]. However, so far, genetic engineering of diatoms has been slow due to the lack of genetic transformation systems, and successful trials of genetically modified diatoms have been rarely reported.

With more and more sequenced genomes, certain metabolic pathways in the corresponding species can be readily proposed. By mapping annotated diatom genes to KEGG (Kyoto encyclopedia of genes and genomes) and taking the reported pathways into account [68,69], the PUFA biosynthetic pathway can be proposed in the diatoms $P$. tricornutum and $T$. pseudonana whose genome sequences are available (Figure 1). Those key points in the pathway are consequently the putative candidates for genetic engineering, as evidenced by studies in various organisms. By introducing a gene encoding a glucose transporter, transgenic $P$. tricornutum could thrive on exogenous glucose without light, thereby becoming heterotrophic [67]. This suggests that introduction of a single gene can lead to a fundamental change in the metabolism of a diatom.

Among the 11 open reading frames (ORFs) with significant similarity to fatty acid front-end desaturases in the genome sequence of T. pseudonana, two members are homologous to proteobacterial desaturases; one encodes a $\Delta 11$-desaturase active on palmitic acid; three encodes $\Delta 4-, \Delta 5-$ and $\Delta 6$-desaturases involved in DHA production; and one encodes a $\Delta 8$-sphingolipid desaturase with substrate preference for dihydroxylated metabolites [70]. Heterologous expression of two P. tricornutum cDNAs encoding a microsomal and a plastidial $\Delta 12$-desaturase in yeast showed that the microsomal desaturase was suggested to be involved in the synthesis of EPA in the microsomes, while the plastidial desaturase showed high specificity for $16: 1^{\Delta 9}$, indicating the plastidial origin of the hexadecatrienoic acid isomer $\left(16: 3^{\Delta 6,9,12}\right)$ in $P$. tricornutum [71]. $\Delta 5$ - and $\Delta 6$-fatty acid desaturases of $P$. tricornutum were characterized by expression in yeast, and the determination of substrate specificity of the two enzymes confirmed their role in EPA biosynthesis. Coexpression of both desaturases in combination with $\Delta 6$-elongase from a moss Physcomitrella patens reconstituted the biosynthetic pathways of arachidonic 
acid (AA) and EPA in yeast [72]. Bi-functional desaturases, such as $\Delta 12 / \Delta 15$-desaturase from the amoebae Acanthamoeba castellanii and the fungus Fusarium moniliforme, have shown significant potential in increasing the productivity of $n-3$ substrates through redirection of the PUFA biosynthetic pathway $[73,74]$. It is worth noting that $n-3$ fatty acid desaturases are also functional enzymes in the synthesis of $n$-3 PUFAs, considering their roles for efficient conversion of $n$ - 6 PUFAs to $n-3$ PUFAs.

Figure 1. Proposed pathway of PUFA synthesis in diatoms.

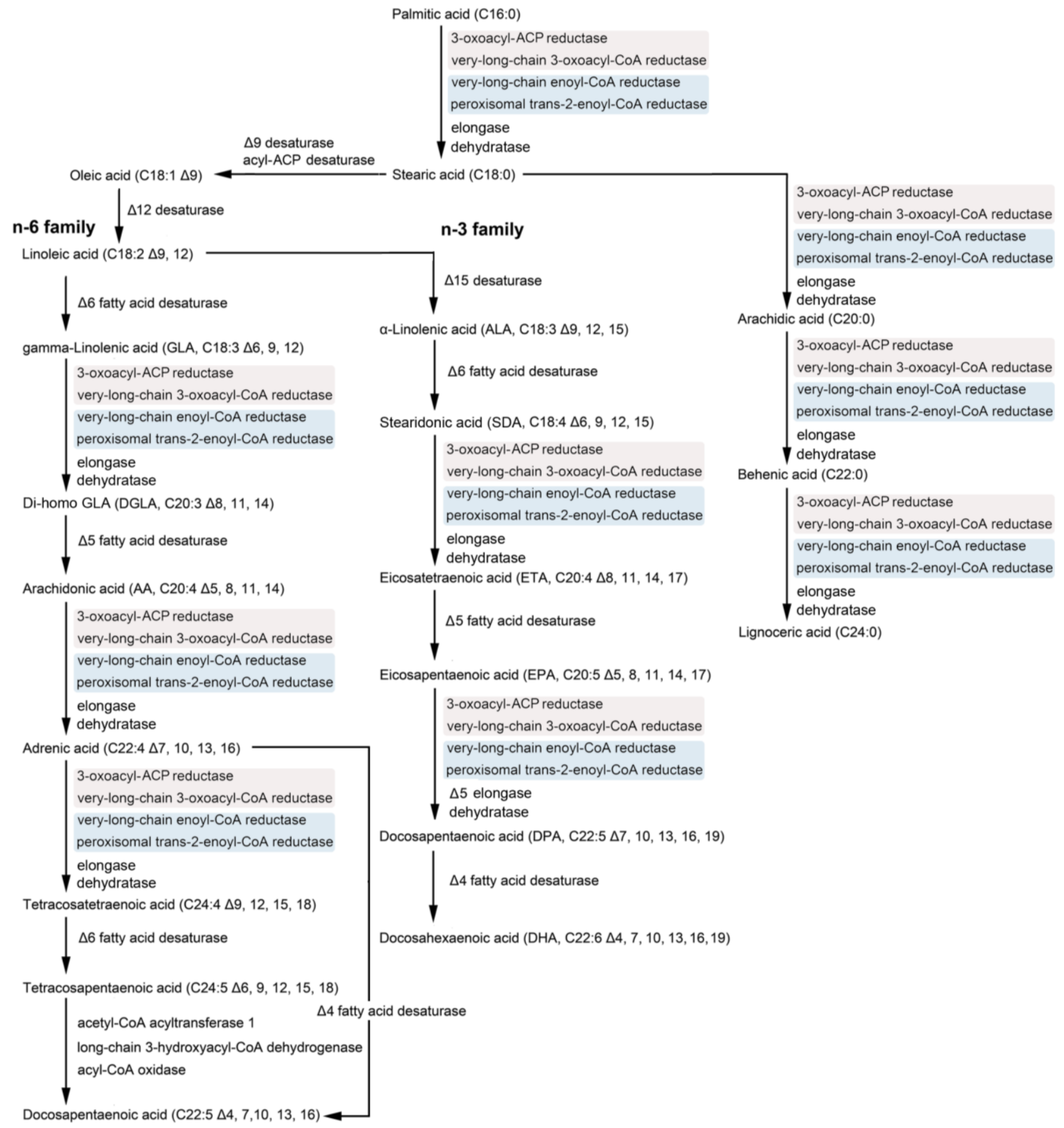

The pathway was proposed by mapping annotated genes to KEGG pathways based on the genomes of diatoms, including P. tricornutum and T. pseudonana. Isoenzymes are highlighted with the same color. 
By heterologous coexpression of the $\Delta 6$-elongase from diatom $T$. pseudonana with $\Delta 5$-elongase, $\Delta 5$ and $\Delta 4$-desaturases from three other algae, DHA synthesis was successfully reconstituted in stearidonic acid-fed yeast [75]. PUFA biosynthesis could also be improved through the regulation of genes involved in lipid metabolism, for instance, overexpression of a Type 2 diacylglycerol acyltransferase which plays an important role in TAG assembly resulted in a 76\% increase in PUFA content in P. tricornutum [76]. Moreover, fatty acid chain length could be regulated by genetic modification on certain genes in diatoms. Heterologous expression of plant acyl-ACP thioesterases biased towards the synthesis of lauric acid (C12:0) and myristic acid (C14:0) in P. tricornutum led to an increased accumulation of shorter chain length fatty acids [77]. Owing to the progress of functional genomics research together with the development and availability of molecular tools for diatoms [78-80], genetically engineering has been providing an effective way to improve the PUFA production phenotype of diatoms.

\section{Conclusions}

The large demand for health-benefiting PUFAs cannot be supplied by the current fish harvest. Therefore, new alternative sources of PUFAs have to be exploited. Using diatoms to produce PUFAs has several advantages, such as controlled culture conditions, lack of contamination, and the presence of PUFAs in the polar lipid fraction along with carotenoids, phytosterols, vitamins and antioxidants, which may also contribute to the health benefits of PUFA oils. Unfortunately, the production cost of autotrophic diatoms is still rather high. Nonetheless, further research is needed to create diatom strains with enhanced productivity of PUFAs. Enhancing the level of PUFAs is a major challenge, but reducing the $n-6$ and $n-3$ metabolic intermediates represents another important issue [65]. Genetically engineered diatoms that may be heterotrophically cultivated provide a new opportunity. Other limiting factors must also be taken into account, such as light, temperature, nutrients (iron, nitrogen, and silicon), $\mathrm{pH}$, etc. for efficient industrial production of PUFA in diatoms.

\section{Acknowledgements}

This work was supported by the National Science and Technology Support Program (2011BAD14B03), Science and Technology Project of Guangdong (2010B030600005, 2009B050600005), and the Fundamental Research Funds for the Central Universities (21610103).

\section{Conflicts of Interest}

The authors declare no conflict of interest.

\section{References}

1. Caldwell, G.S. The Influence of Bioactive Oxylipins from marine diatoms on invertebrate reproduction and development. Mar. Drugs 2009, 7, 367-400.

2. Guo, S.L.; Zhao, X.Q.; Tang, Y.; Wan, C.; Alam, M.A.; Ho, S.H.; Bai, F.W.; Chang, J.S. Establishment of an efficient genetic transformation system in Scenedesmus obliquus. J. Biotechnol. 2013, 163, 61-68. 
3. Falkowski, P.G.; Barber, R.T.; Smetacek, V. Biogeochemical controls and feedbacks on ocean primary production. Science 1998, 281, 200-206.

4. Armbrust, E.V.; Berges, J.A.; Bowler, C.; Green, B.R.; Martinez, D.; Putnam, N.H.; Zhou, S.; Allen, A.E.; Apt, K.E.; Bechner, M.; et al. The genome of the diatom Thalassiosira pseudonana: Ecology, evolution, and metabolism. Science 2004, 306, 79-86.

5. Schmaljohann, R.; Röttger, R. The ultrastructure and taxonomic identity of the symbiotic algae of Heterostegina depressa (Foraminifera, Nummulitidae). J. Mar. Biol. Assoc. UK 1978, 58, 227-237.

6. Desbois, A.P.; Mearns-Spragg, A.; Smith, V.J. A fatty acid from the diatom Phaeodactylum tricornutum is antibacterial against diverse bacteria including multi-resistant Staphylococcus aureus (MRSA). Mar. Biotechnol. 2009, 11, 45-52.

7. Norton, T.A.; Melkonian, M.; Andersen, R.A. Algal biodiversity. Phycologia 1996, 35, 308-326.

8. Hamm, C.E.; Merkel, R.; Springer, O.; Jurkojc, P.; Maier, C.; Prechtel, K.; Smetacek, V. Architecture and material properties of diatom shells provide effective mechanical protection. Nature 2003, 421, 841-843.

9. Peng, J.; Yuan, J.P.; Wu, C.F.; Wang, J.H. Fucoxanthin, a marine carotenoid present in brown seaweeds and diatoms: Metabolism and bioactivities relevant to human health. Mar. Drugs 2011, 9 , $1806-1828$.

10. Schmidt, E.B.; Christensen, J.H.; Aardestrup, I.; Madsen, T.; Riahi, S.; Hansen, V.E.; Skou, H.A. Marine n-3 fatty acids: Basic features and background. Lipids 2001, 36, S65-S68.

11. Sapieha, P.; Stahl, A.; Chen, J.; Seaward, M.R.; Willett, K.L.; Krah, N.M.; Dennison, R.J.; Connor, K.M.; Aderman, C.M.; Liclican, E. 5-Lipoxygenase metabolite 4-HDHA is a mediator of the antiangiogenic effect of $\omega-3$ polyunsaturated fatty acids. Sci. Transl. Med. 2011, 3, 69ra12.

12. Hallahan, B.; Garland, M.R. Essential fatty acids and mental health. Br. J. Psychiatry 2005, 186, $275-277$.

13. Lafourcade, M.; Larrieu, T.; Mato, S.; Duffaud, A.; Sepers, M.; Matias, I.; De Smedt-Peyrusse, V.; Labrousse, V.F.; Bretillon, L.; Matute, C. Nutritional omega-3 deficiency abolishes endocannabinoid-mediated neuronal functions. Nature Neurosci. 2011, 14, 345-350.

14. Lands, W.E. Human life: caught in the food web. In Lipids in Aquatic Ecosystems; Springer: Dordrecht, The Netherlands, 2009; pp. 327-354.

15. Simopoulos, A.P. The importance of the omega-6/omega-3 fatty acid ratio in cardiovascular disease and other chronic diseases. Exp. Biol. Med. 2008, 233, 674-688.

16. Martins, D.A.; Custodio, L.; Barreira, L.; Pereira, H.; Ben-Hamadou, R.; Varela, J.; Abu-Salah, K.M. Alternative Sources of $n-3$ long-chain polyunsaturated fatty acids in marine microalgae. Mar. Drugs 2013, 11, 2259-2281.

17. Valenzuela, R.; Sanhueza, J.; Valenzuela, A. Docosahexaenoic Acid (DHA), an Important Fatty Acid in Aging and the Protection of Neurodegenerative Diseases. J. Nutr. Ther. 2012, 1, 63-72.

18. SanGiovanni, J.P.; Parra-Cabrera, S.; Colditz, G.A.; Berkey, C.S.; Dwyer, J.T. Meta-analysis of dietary essential fatty acids and long-chain polyunsaturated fatty acids as they relate to visual resolution acuity in healthy preterm infants. Pediatrics 2000, 105, 1292-1298.

19. Brett, M.; Müller-Navarra, D. The role of highly unsaturated fatty acids in aquatic foodweb processes. Freshw. Biol. 1997, 38, 483-499. 
20. Arts, M.T.; Kohler, C.C. Health and condition in fish: the influence of lipids on membrane competency and immune response. In Lipids in Aquatic Ecosystems; Springer: Dordrecht, The Netherlands, 2009; pp. 237-256.

21. Tocher, D.R. Metabolism and functions of lipids and fatty acids in teleost fish. Rev. Fish. Sci. 2003, 11, 107-184.

22. Gerber, L.R.; Karimi, R.; Fitzgerald, T.P. Sustaining seafood for public health. Front. Ecol. Environ. 2012, 10, 487-493.

23. Meyer, B.J.; Mann, N.J.; Lewis, J.L.; Milligan, G.C.; Sinclair, A.J.; Howe, P.R. Dietary intakes and food sources of omega-6 and omega-3 polyunsaturated fatty acids. Lipids 2003, 38, 391-398.

24. Sayanova, O.; Napier, J.A. Transgenic oilseed crops as an alternative to fish oils. Prostaglandins Leukot. Essent. Fatty Acids 2011, 85, 253-260.

25. Hu, Q.; Sommerfeld, M.; Jarvis, E.; Ghirardi, M.; Posewitz, M.; Seibert, M.; Darzins, A. Microalgal triacylglycerols as feedstocks for biofuel production: perspectives and advances. Plant J. 2008, 54, 621-639.

26. Lebeau, T.; Robert, J.M. Diatom cultivation and biotechnologically relevant products. Part I: Cultivation at various scales. Appl. Microbiol. Biotechnol. 2003, 60, 612-623.

27. Belarbi, E.-H.; Molina, E.; Chisti, Y. RETRACTED: A process for high yield and scaleable recovery of high purity eicosapentaenoic acid esters from microalgae and fish oil. Process Biochem. 2000, 35, 951-969.

28. Lavens, P.; Sorgeloos, P. Manual on the Production and Use of Live Food for Aquaculture; Food and Agriculture Organization (FAO): Rome, Italy, 1996.

29. Pulz, O.; Scheibenbogen, K. Photobioreactors: design and performance with respect to light energy input. In Bioprocess and Algae Reactor Technology, Apoptosis; Springer: Dordrecht, The Netherlands, 1998; pp. 123-152.

30. Fernández, F.; Pérez, J.; Sevilla, J.; Camacho, F.G.; Grima, E.M. Modeling of eicosapentaenoic acid (EPA) production from Phaeodactylum tricornutum cultures in tubular photobioreactors. Effects of dilution rate, tube diameter, and solar irradiance. Biotechnol. Bioeng. 2000, 68, 173-183.

31. Piepho, M.; Arts, M.T.; Wacker, A. Species-specific variation in fatty acid concentrations of four phytoplankton species: Does phosphorus supply influence the effect of light intensity or temperature? J. Phycol. 2012, 48, 64-73.

32. Meiser, A.; Schmid-Staiger, U.; Trösch, W. Optimization of eicosapentaenoic acid production by Phaeodactylum tricornutum in the flat panel airlift (FPA) reactor. J. Appl. Phycol. 2004, 16, 215-225.

33. Hill, W.R.; Rinchard, J.; Czesny, S. Light, nutrients and the fatty acid composition of stream periphyton. Freshw. Biol. 2011, 56, 1825-1836.

34. Renaud, S.; Zhou, H.; Parry, D.; Thinh, L.-V.; Woo, K. Effect of temperature on the growth, total lipid content and fatty acid composition of recently isolated tropical microalgae Isochrysis sp., Nitzschia closterium, Nitzschia paleacea, and commercial species Isochrysis sp.(clone T. ISO). J. Appl. Phycol. 1995, 7, 595-602.

35. Thompson, P.A.; Guo, M.X.; Harrison, P.J. Effects of variation in temperature. I. On the biochemical composition of eight species of marine phytoplankton. J. Phycol. 1992, 28, 481-488. 
36. James, C.; Al-Hinty, S.; Salman, A. Growth and $\omega 3$ fatty acid and amino acid composition of microalgae under different temperature regimes. Aquaculture 1989, 77, 337-351.

37. Kudo, I.; Miyamoto, M.; Noiri, Y.; Maita, Y. Combined effects of temperature and iron on the growth and physiology of the marine diatom Phaeodactylum tricornutum (Bacillariophyceae). J. Phycol. 2000, 36, 1096-1102.

38. Scholz, B.; Liebezeit, G. Compatible solutes and fatty acid composition of five marine intertidal microphytobenthic Wadden Sea diatoms exposed to different temperature regimes. Diatom Res. 2013, 28, 337-358.

39. Harwood, J.L. Fatty acid metabolism. Ann. Rev. Plant Physiol. Plant Mol. Biol. 1988, 39, 101-138.

40. Jiang, H.; Gao, K. Effects of lowering temperature during culture on the production of polyunsaturated Fatty Acids in the Marine Diatom Phaeodactylum tricornutum (Bacillariophyceae)1. J. Phycol. 2004, 40, 651-654.

41. Paasche, E. Silicon and the ecology of marine plankton diatoms. I. Thalassiosira pseudonana (Cyclotella nana) grown in a chemostat with silicate as limiting nutrient. Mar. Biol. 1973, 19, 117-126.

42. Moore, J.K.; Doney, S.C.; Glover, D.M.; Fung, I.Y. Iron cycling and nutrient-limitation patterns in surface waters of the World Ocean. Deep Sea Res. II Topical. Stud. Oceanogr. 2001, 49, 463-507.

43. Dortch, Q. The interaction between ammonium and nitrate uptake in phytoplankton. Mar. Ecol. Prog. Ser. 1990, 61, 183-201.

44. Bender, S.J.; Parker, M.S.; Armbrust, E. Coupled effects of light and nitrogen source on the urea cycle and nitrogen metabolism over a diel cycle in the marine diatom Thalassiosira pseudonana. Protist 2012, 163, 232-251.

45. Suman, K.; Kiran, T.; Devi, U.K.; Sarma, N.S. Culture medium optimization and lipid profiling of Cylindrotheca, a lipid- and polyunsaturated fatty acid-rich pennate diatom and potential source of eicosapentaenoic acid. Bot. Mar. 2012, 55, 289-299.

46. Miller, R.; Wu, G.; Deshpande, R.R.; Vieler, A.; Gartner, K.; Li, X.; Moellering, E.R.; Zauner, S.; Cornish, A.J.; Liu, B.; et al. Changes in transcript abundance in Chlamydomonas reinhardtii following nitrogen deprivation predict diversion of metabolism. Plant Physiol. 2010, 154, 1737-1752.

47. Illman, A.; Scragg, A.; Shales, S. Increase in Chlorella strains calorific values when grown in low nitrogen medium. Enzym. Microb. Technol. 2000, 27, 631-635.

48. Qian, K.; Michael, A.B. Light and nitrogen deficiency effects on the growth and composition of Phaeodactylum tricornutum. Appl. Biochem. Botechnol. 1993, 38, 93-103.

49. Zhila, N.O.; Kalacheva, G.S.; Volova, T.G. Influence of nitrogen deficiency on biochemical composition of the green alga Botryococcus. J. Appl. Phycol. 2005, 17, 309-315.

50. Davidi, L.; Katz, A.; Pick, U. Characterization of major lipid droplet proteins from Dunaliella. Planta 2012, 236, 19-33.

51. Yang, Z.-K.; Ma, Y.-H.; Zheng, J.-W.; Yang, W.-D.; Liu, J.-S.; Li, H.-Y. Molecular and cellular mechanisms of neutral lipid accumulation in diatom following nitrogen deprivation. Biotechnol. Biofuels 2013, 6, 67:1-67:14. 
52. Milligan, A.J.; Harrison, P.J. Effects of non-steady-state iron limitation on nitrogen assimilatory enzymes in the marine diatom Thalassiosira weissflogii (Bacillariophyceae). J. Phycol. 2000, 36, $78-86$.

53. Renaud, S.M.; Thinh, L.-V.; Parry, D.L. The gross chemical composition and fatty acid composition of 18 species of tropical Australian microalgae for possible use in mariculture. Aquaculture 1999, 170, 147-159.

54. Bertrand, E.M.; Allen, A.; Dupont, C.L.; Norden-Krichmar, T.M.; Bai, J.; Valas, R.E.; Saito, M.A. Influence of cobalamin scarcity on diatom molecular physiology and identification of a cobalamin acquisition protein. Proc. Natl. Acad. Sci. USA 2012, 109, E1762-E1771.

55. Mortensen, S.H.; Børsheim, K.Y.; Rainuzzo, J.; Knutsen, G. Fatty acid and elemental composition of the marine diatom Chaetoceros gracilis Schütt. Effects of silicate deprivation, temperature and light intensity. J. Exp. Mar. Biol. Ecol. 1988, 122, 173-185.

56. Kates, M.; Volcani, B. Lipid components of diatoms. Biochim. Biophys. Acta 1966, 116, 264-278.

57. Ying, L.; Kang-sen, M.; Shi-chun, S. Effects of harvest stage on the total lipid and fatty acid composition of four Cylindrotheca strains. Chin. J. Ocean. Limnol. 2002, 20, 157-161.

58. Brown, M.R.; Dunstan, G.A.; Norwood, S.; Miller, K.A. Effects of harvest stage and light on the biochemical composition of the diatom Thalassiosira peudonana. J. Phycol. 1996, 32, 64-73.

59. Ugwu, C.U.; Aoyagi, H.; Uchiyama, H. Photobioreactors for mass cultivation of algae. Bioresour. Technol. 2008, 99, 4021-4028.

60. Molina, E.; Fernández, J.; Acién, F.G.; Chisti, Y. Tubular photobioreactor design for algal cultures. J. Biotechnol. 2001, 92, 113-131.

61. Grima, E.M.; FernaÂndez, F.G.A.; Camacho, F.G.; Chisti, Y. Photobioreactors: light regime, mass transfer, and scaleup. J. Biotechnol. 1999, 70, 231-247.

62. Weissman, J.C.; Goebel, R.P.; Benemann, J.R. Photobioreactor design: Mixing, carbon utilization, and oxygen accumulation. Biotechnol. Bioeng. 1988, 31, 336-344.

63. Mata, T.M.; Martins, A.A.; Caetano, N.S. Microalgae for biodiesel production and other applications: A review. Renew. Sustain. Energy Rev. 2010, 14, 217-232.

64. Chen, C.Y.; Yeh, K.L.; Aisyah, R.; Lee, D.J.; Chang, J.S. Cultivation, photobioreactor design and harvesting of microalgae for biodiesel production: A critical review. Bioresour. Technol. 2011, 102, 71-81.

65. Ryckebosch, E.; Bruneel, C.; Muylaert, K.; Foubert, I. Microalgae as an alternative source of omega-3 long chain polyunsaturated fatty acids. Lipid Technol. 2012, 24, 128-130.

66. Wu, X.; Ouyang, H.; Duan, B.; Pang, D.; Zhang, L.; Yuan, T.; Xue, L.; Ni, D.; Cheng, L.; Dong, S.; et al. Production of cloned transgenic cow expressing omega-3 fatty acids. Transgenic Res. 2012, 21, 537-543.

67. Zaslavskaia, L.A.; Lippmeier, J.C.; Shih, C.; Ehrhardt, D.; Grossman, A.R.; Apt, K.E. Trophic conversion of an obligate photoautotrophic organism through metabolic engineering. Science 2001, 292, 2073-2075.

68. Khozin-Goldberg, I.; Iskandarov, U.; Cohen, Z. LC-PUFA from photosynthetic microalgae: Occurrence, biosynthesis, and prospects in biotechnology. Appl. Microbiol. Biotechnol. 2011, 91, 905-915. 
69. Haslam, R.P.; Ruiz-Lopez, N.; Eastmond, P.; Moloney, M.; Sayanova, O.; Napier, J.A. The modification of plant oil composition via metabolic engineering - better nutrition by design. Plant Biotechnol. J. 2013, 11, 157-168.

70. Tonon, T.; Sayanova, O.; Michaelson, L.V.; Qing, R.; Harvey, D.; Larson, T.R.; Li, Y.; Napier, J.A.; Graham, I.A. Fatty acid desaturases from the microalga Thalassiosira pseudonana. FEBS J. 2005, 272, 3401-3412.

71. Domergue, F. New Insight into Phaeodactylum tricornutum Fatty Acid Metabolism. Cloning and Functional Characterization of Plastidial and Microsomal Delta 12-Fatty Acid Desaturases. Plant Physiol. 2003, 131, 1648-1660.

72. Domergue, F.; Lerchl, J.; Zähringer, U.; Heinz, E. Cloning and functional characterization of Phaeodactylum tricornutum front-end desaturases involved in eicosapentaenoic acid biosynthesis. Eur. J. Biochem. 2002, 269, 4105-4113.

73. Damude, H.G.; Zhang, H.; Farrall, L.; Ripp, K.G.; Tomb, J.-F.; Hollerbach, D.; Yadav, N.S. Identification of bifunctional $\Delta 12 / \omega 3$ fatty acid desaturases for improving the ratio of $\omega 3$ to $\omega 6$ fatty acids in microbes and plants. Proc. Natl. Acad. Sci. USA 2006, 103, 9446-9451.

74. Sayanova, O.; Haslam, R.; Guschina, I.; Lloyd, D.; Christie, W.W.; Harwood, J.L.; Napier, J.A. A bifunctional $\Delta 12, \Delta 15$-desaturase from Acanthamoeba castellanii directs the synthesis of highly unusual n-1 series unsaturated fatty acids. J. Biol. Chem. 2006, 281, 36533-36541.

75. Meyer, A.; Kirsch, H.; Domergue, F.; Abbadi, A.; Sperling, P.; Bauer, J.; Cirpus, P.; Zank, T.K.; Moreau, H.; Roscoe, T.J. Novel fatty acid elongases and their use for the reconstitution of docosahexaenoic acid biosynthesis. J. Lipid Res. 2004, 45, 1899-1909.

76. Niu, Y.-F.; Zhang, M.-H.; Li, D.-W.; Yang, W.-D.; Liu, J.-Sheng.; Bai, W.-B.; Li, H.-Y. Improvement of neutral lipid and polyunsaturated fatty acid biosynthesis by overexpressing a type 2 diacylglycerol acyltransferase in marine diatom Phaeodactylum tricornutum. Mar. Drugs 2013, $11,4558-4569$.

77. Radakovits, R.; Eduafo, P.M.; Posewitz, M.C. Genetic engineering of fatty acid chain length in Phaeodactylum tricornutum. Metab. Eng. 2011, 13, 89-95.

78. Norden-Krichmar, T.M.; Allen, A.E.; Gaasterland, T.; Hildebrand, M. Characterization of the small RNA transcriptome of the diatom, Thalassiosira pseudonana. PLoS One 2011, 6, e22870.

79. Valenzuela, J.; Mazurie, A.; Carlson, R.P.; Gerlach, R.; Cooksey, K.E.; Peyton, B.M.; Fields, M.W. Potential role of multiple carbon fixation pathways during lipid accumulation in Phaeodactylum tricornutum. Biotechnol. Biofuels 2012, 5, 1-17.

80. Siaut, M.; Heijde, M.; Mangogna, M.; Montsant, A.; Coesel, S.; Allen, A.; Manfredonia, A.; Falciatore, A.; Bowler, C. Molecular toolbox for studying diatom biology in Phaeodactylum tricornutum. Gene 2007, 406, 23-35.

(C) 2014 by the authors; licensee MDPI, Basel, Switzerland. This article is an open access article distributed under the terms and conditions of the Creative Commons Attribution license (http://creativecommons.org/licenses/by/3.0/). 